\title{
Ex vivo cardiovascular magnetic resonance measurements of right and left ventricular mass compared with direct mass measurement in excised hearts after transplantation: a first human SSFP comparison
}

Nicholas J Farber ${ }^{1}$, Sahadev T Reddy ${ }^{1}$, Mark Doyle1, Geetha Rayarao ${ }^{1}$, Diane V Thompson ${ }^{1}$, Peter Olson², Jerry Glass ${ }^{2}$, Ronald B Williams ${ }^{1}$, June A Yamrozik', Srinivas Murali ${ }^{1}$ and Robert WW Biederman ${ }^{\text {* }}$

\begin{abstract}
Background: CMR is considered the 'gold standard' for non-invasive LV and RV mass quantitation. This information is solely based on gradient-recalled echo (GRE) sequences while contrast dependent on intrinsic $\mathrm{T} 1 / \mathrm{T} 2$ characteristics potentially offers superior image contrast between blood and myocardium. This study aims, for the first time in humans, to validate the SSFP approach using explanted hearts obtained from heart transplant recipients. Our objective is establish the correlation between and to validate steady-state free precession (SSFP) derived LV and RV mass Vs. autopsy mass of hearts from cardiac transplants patients.

Methods: Over three-years, 58 explanted cardiomyopathy hearts were obtained immediately upon orthotopic heart transplantation from the OR. They were quickly cleaned, prepared and suspended in a saline-filled container and scanned ex vivo via SSFP-SA slices to define LV/RV mass. Using an automatic thresholding program, segmentation was achieved in combination with manual trimming (ATMT) of extraneous tissue incorporating 3D cardiac modeling performed by independent and blinded readers. The explanted hearts were then dissected with the ventricles surgically separated at the interventricular septum. Weights of the total heart not excluding papillary and trabecular myocardium, LV and RV were measured via high-fidelity scale. Linear regression and Bland-Altman plots were used to analyze the data. The intra-class correlation coefficient was used to assess intra-observer reliability.
\end{abstract}

Results: Of the total of 58 explanted hearts, 3 (6\%) were excluded due to poor image quality leaving 55 patients (94\%) for the final analysis. Significant positive correlations were found between total 3D CMR mass $(450 \pm 111 \mathrm{~g})$ and total pathology mass ( $445 \pm 116 \mathrm{~g} ; \mathrm{r}=0.99, \mathrm{p}<0.001)$ as well as 3D CMR measured LV mass (301 $\pm 93 \mathrm{~g})$ and the pathology measured LV mass (313 $\pm 96 \mathrm{~g} ; r=0.95, p<0.001)$. Strong positive correlations were demonstrated between the 3D CMR measured RV mass $(149 \pm 46 \mathrm{~g})$ and the pathology measured RV mass $(128 \pm 40 \mathrm{~g} ; \mathrm{r}=0.76, \mathrm{p}<0.001)$. The mean bias between 3D-CMR and pathology measures for total mass, LV mass and RV mass were: $3.0 \mathrm{~g},-16 \mathrm{~g}$ and $19 \mathrm{~g}$, respectively. (Continued on next page)

\footnotetext{
* Correspondence: RBIEDERM@wpahs.org

'Gerald McGinnis Cardiovascular Institute, Allegheny General Hospital, Pittsburgh, PA, USA

Full list of author information is available at the end of the article
} 
(Continued from previous page)

Conclusions: SSFP-CMR accurately determines total myocardial, LV and RV mass as compared to pathology weighed explanted hearts despite variable surgical removal of instrumentation (left and right ventricular assist devices, AICD and often apical core removals). Thus, this becomes the first-ever human CMR confirmation for SSFP now validating the distinction of 'gold standard'.

Keywords: Cardiovascular magnetic resonance, Cardiac mass, LV and RV mass, Explanted hearts, Steady-state free precession

\section{Background}

An accurate and reproducible measurement of both left ventricular mass (LVM) and right ventricular mass (RVM) is important for cardiac risk stratification. Currently, cardiovascular magnetic resonance (CMR) imaging is considered the 'gold standard' for non-invasive measurement of left and right ventricular volume, mass and by extension LVEF and RVEF [1,2]. CMR offers a spatially defined 3D data set that spans the entire heart. The volume and mass measurements afforded by CMR are thus independent of cardiac geometric assumptions and are a major advantage over $1 \mathrm{D}$ and 2D echocardiography. This issue is particularly important for the RV, as its complex, crescentic geometry is challenging for mathematical modeling, placing increased importance on accurate mass calculations free of geometric assumptions [3]. A variety of prior studies have demonstrated CMR's superior accuracy and reproducibility in assessing ventricular volumes over other imaging modalities, including $\mathrm{M}$-mode and 2D echocardiography [4-9]. While the assessment of left ventricular mass by real-time 3D echocardiography has overcome some of the geometric assumptions required of 2D echocardiography methods, its clinical utility is still limited when characterizing the right ventricle and in patients with a poor acoustic window [10].

An ideal way to assess the mass calculation capabilities and intrinsic accuracy of any imaging modality is to independently compare the imaging-derived mass to a corresponding autopsy mass. In this way Devereux and Reichek in 1977 performed their seminal work comparing echocardiography with autopsy validation to derive an imaging-based left ventricular mass equation [11]. Their approach is closely replicated herein for the first time with CMR. Briefly, their intrepid work involved identifying terminally ill patients and obtaining research echocardiograms prior to autopsy, then comparing these in vivo ventricular mass measurements to the autopsy measurements. Before then, the determination of echocardiographic LV mass calculations was only postulated, not validated by autopsy. Currently, the recognition of CMR as the gold standard for non-invasive left and right ventricular mass calculation is largely based on animal and phantom data; the only human autopsy studies, as explained below, do not utilize the most recent pulse sequences, undermining the credibility of CMR as the true 'gold standard' [12-15]. Moreover, these older studies, in addition to small sample sizes, employed spinecho or gradient recalled echo (GRE) pulse sequences for image acquisition. GRE sequences had numerous well defined deficiencies including flow-related enhancement and reliance on flow to define cardiac boundaries with consequent overestimation of mass and underestimation of volume. Despite these limitations, GRE served remarkably well for several decades. However, as is well known, the newer steady-state free precession (SSFP) approach not only has become the workhorse sequence for CMR due to its intrinsic T1/T2 contrast attributes but has become the de facto gold standard for measurement of LV mass due to its inherent resolution. Thus, there is an obligate need to reassess and validate CMR using SSFP sequences as the current gold standard for ventricular mass quantification.

It should be noted that when validated with animal data, SSFP was shown to be the scanning sequence that yielded CMR-derived ventricular mass closest to the autopsy mass [16]. Thus, the next logical step is to validate mass acquisition by CMR's SSFP sequences in human hearts by autopsy. Therefore, the aim of our study was to establish a correlation between CMR derived left and right ventricular mass and autopsy-derived ventricular mass of ex vivo hearts from heart transplants using the SSFP sequence, thereby determining the credibility of CMR as the gold standard for non-invasive left and right ventricular mass calculation. However, today's ability to replicate the autopsy approach used by Devereux and Reichek is more constrained than in the past as the more restrictive research environment, with increased protection of patients and institutional review boards, makes it all but impossible to duplicate their methods. Nonetheless, there are alternative ways, which are more sensitive to patients' rights and would achieve similar results to what was done in the 1970's, to execute this study. Our solution was to use the diseased hearts (explanted) from orthotopic heart transplantation as the source of hearts for imaging. Naturally, this approach removes the need and arguable issue of in vivo scanning of terminally ill patients just to establish a bona fide gold standard. 


\section{Methods}

In this prospective IRB approved study, explanted hearts from heart transplant recipients $(n=58)$ were obtained at the time of orthotopic transplantation within five minutes of harvest. The hearts were prepared by trimming atria and vascular components leaving only LV and RV myocardium including papillary and trabecular tissue. The hearts were submerged in a saline filled plastic container and CMR imaging was performed (GE, Milwaukee, WI; $1.5 \mathrm{~T}$ ) using standard steady-state-free-precession (SSFP) approach (TR/TE/flip angle 4.2/2.1/45; FOV $350 \mathrm{~mm}$, matrix $256 \times 256$, scan percentage $100 \%$.) with slices oriented parallel to the LV short axis, applied contiguously from base to apex (slice thickness $8 \mathrm{~mm}$, gap $0 \mathrm{~mm}$ ). A birdcage design neurovascular coil was used to receive the signal. Using an image analysis program available with our scanner (Medis, The Netherlands), Automatic Thresholding with Manual Trimming (ATMT) was performed on the CMR set of images to isolate and contour cardiac tissue. Specifically, the ATMT approach was utilized given the complexity of the heart analysis with necessary removal of LVAD and/or RVAD apical cores, lateral wall surgical frozen section removal and AICD/PM lead extractions that markedly and exceptionally non-uniformly destroyed the primary myocardial architecture and geometry so critical for natural and heuristic manual endocardial and epicardial contour analysis. Formally, ATMT involved the automatic setting of an upper and lower myocardial signal threshold such that cardiac tissue was, via signal intensity, isolated from the surrounding saline bath signal from which the heart was suspended for imaging. Secondly, the signal was rendered as a 3D structure, which could be rotated within the viewing station permitting more user-controlled defined ROI contours to the often no-longer intuitive LV/RV geometry. Using a succession of cuts applied to the 3D model, non-cardiac tissue signal (saline which had near identical signal intensity to fat, distortion artifacts as apex approached the container and retained air) was systematically removed leaving a 3D model for the independent and blinded reader facilitating final manual contouring. In short: assisted manual contouring to permit formal comparison of CMR mass to autopsy mass whereas the former is and the latter is not dependent on uniformity of structure (Figure 1).

Initially, the whole cardiac structure was isolated, and using the features of the processing software, the volume of myocardium was determined. Next, this was converted into grams by multiplication of the density of myocardium, taken as $1.055 \mathrm{~g} / \mathrm{mm}^{3}$. After recording the total cardiac volume, the same model was further operated on to remove the RV structure by use of a series of digital 'cuts' applied to the 3D model. The remaining tissue (typically trimmed to a semi elliptical structure) was measured and taken as the LV or RV volume. Following performance of the CMR examination, the explanted hearts were returned to the Pathology Lab, where the whole heart was weighed using a high-fidelity scale. The pathologist then formally separated the RV tissue using scissors, and the RV and LV tissue were weighed separately. These steps understandably required intricate coordination between the transplant surgeons, pathologists and the CMR Laboratory day or night given the natural nuances of obtaining donor hearts. Finally, ten random explant hearts were also reanalyzed to establish the intra-observer variability by the same observer (GR) using the exact same technique. The reanalysis was blinded and took place 4 months after the initial analysis to avoid memory bias.

\section{Statistical analysis}

Continuous variables were expressed as mean + standard deviation and compared using the independent samples $t$ test. Categorical variables were expressed as counts and percentages. Linear regressions were performed to assess the correlation of cardiac mass weights between the SSFPCMR method and ex-vivo heart autopsy method. BlandAltman plots were used to determine the bias and 95\% limits of agreement between methods. A two-tailed p-value $<0.05$ was considered to indicate statistical significance. The intra-class correlation coefficient (ICC) was calculated to assess intra-observer reliability between measures of 3D MRI volumes made by a single rater using the ATMT method. The ICC model used was two-way mixed and the type was absolute agreement. The values of ICC range between 0 and 1.0, with values closer to 1.0 representing stronger reliability. Portney and Watkins [17] suggested that ICC value above 0.75 indicate good reliability, those between 0.5 and 0.75 indicate moderate reliability, and those below .50 indicate poor reliability). Data was analyzed using IBM SPSS Statistics, version 20.0 (IBM Corporation, Armonk, NY).

\section{Results}

A total of 58 explanted hearts were imaged; 3 (5\%) were excluded due to poor image quality (peri-surgical destruction) leaving 55 (95\%) for the final analysis. Of the 55 explanted hearts, 39 (71\%) were men, and 16 (29\%) were women (mean age $55 \pm 11$ years, range 23 to 73 years). A significant proportion of the explanted hearts belonged to ischemic (47\%) and non-ischemic heart disease (36\%) patients (Table 1). Instrumentation was present in all but two patients comprised of AICD and or PM (93\%), LVAD (60\%), RVAD (15\%) and/or RVAD/LVAD (12\%). All (100\%) patients had LV and RV (42\%) myocardial surgical resection of varying amounts averaging $3 \times 3 \mathrm{~cm}$. The average time from Transplant Suite to Pathology Department was $25 \pm 5$ and from Pathology to CMR notification was $30 \pm 5$ minutes. CMR preparation time (RB or $R W)$ was 
a

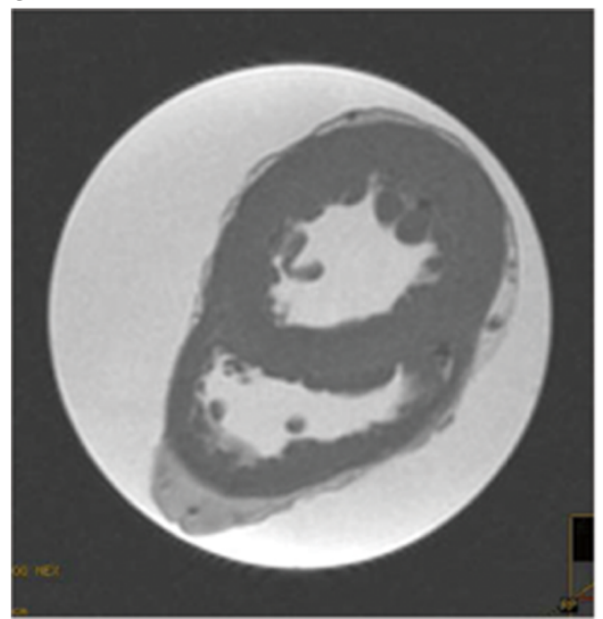

C

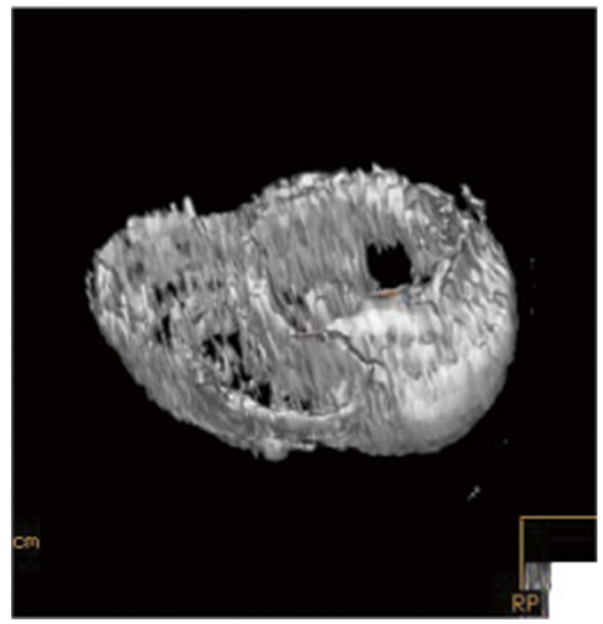

b

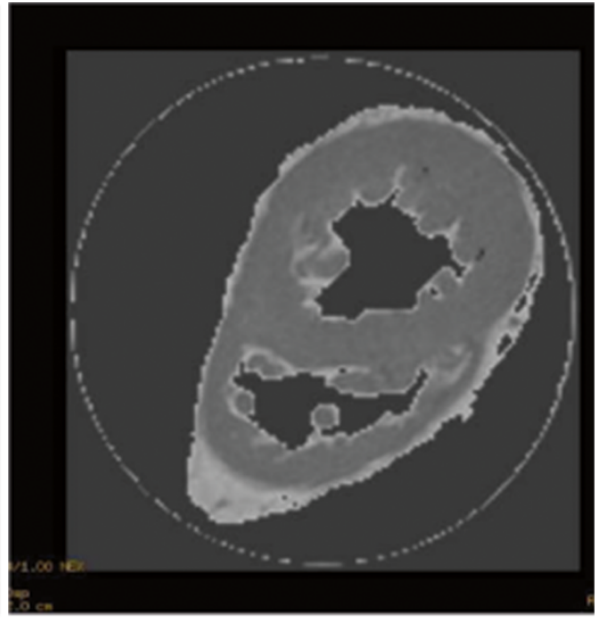

d

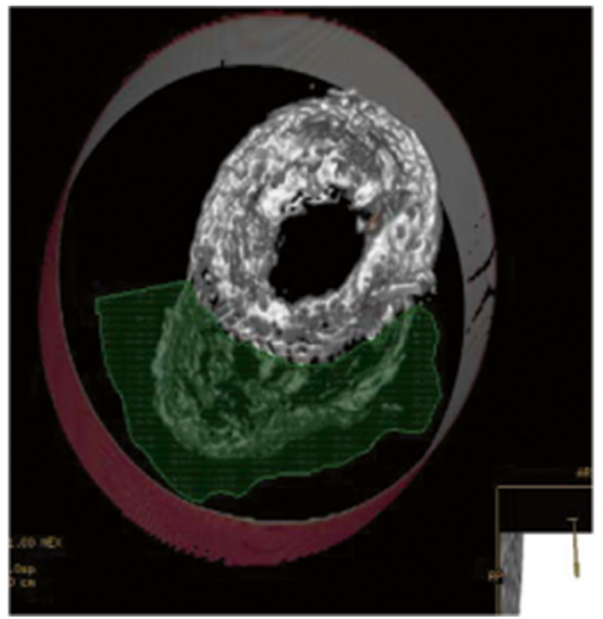

Figure 1 Summary of ATMT steps. a) Cross-sectional view of an explanted heart submerged in a cylindrical water bath. c) Application of upper and lower intensity thresholds to eliminate bulk of signal associated with the water bath. c) By successively removing extra-cardiac features, the 3D heart voxels are isolated. $\mathbf{d}$ ) To isolate the left ventricle an irregular region encompassing the right ventricle is manually identified for removal.

$10 \pm 2$ minutes minimizing desiccation and impending formaldehyde preservation. The processing time per image series was approximately 8-10 min; not significantly different than standard manual contouring. The total time the hearts were rerouted to the CMR facility was $<60$ minutes.

Extremely strong positive correlations between total CMR 3D mass $(450 \pm 111 \mathrm{~g})$ and total pathology mass $(445 \pm 116 \mathrm{~g} ; \mathrm{r}=0.99, \mathrm{p}<0.001)$ and the $3 \mathrm{D} \mathrm{CMR}$ measured LV mass (301 $\pm 93 \mathrm{~g})$ versus the pathology measured LV mass $(313 \pm 96$ g; $r=0.95, p<0.001)$. Strong positive correlation was demonstrated between the $3 \mathrm{D}$ CMR measured RV mass $(149 \pm 46 \mathrm{~g})$ and the pathology measured RV mass $(128 \pm 40 \mathrm{~g} ; \mathrm{r}=0.77, \mathrm{p}<0.001) \mathrm{R}=0.77$ (Table 2). The equations $\mathrm{y}=1.023 \times-15.2 \mathrm{~b}(\mathrm{r}=0.99) ; \mathrm{y}=$

\section{Table 1 Heart transplant demographics}

\begin{tabular}{ll}
\hline Demographics & Number (55) \\
\hline Age (years) & $55 \pm 11$ \\
Female & $16(29 \%)$ \\
Male & $39(71 \%)$ \\
Ischemic Cardiomyopathy & $26(47 \%)$ \\
Hypertrophic Cardiomyopathy & $2(4 \%)$ \\
Miscellaneous Etiologies & $3(6 \%)$ \\
Non-ischemic Cardiomyopathy & $20(36 \%)$ \\
Re-transplantation & $4(7 \%)$ \\
\hline
\end{tabular}


Table 2 Cardiac mass weight cmr vs. pathology

\begin{tabular}{llll}
\hline Parameter & $\begin{array}{l}\text { Total cardiac } \\
\text { mass (g) }\end{array}$ & $\begin{array}{l}\text { Left ventricular } \\
\text { mass (g) }\end{array}$ & $\begin{array}{l}\text { Right ventricular } \\
\text { mass (g) }\end{array}$ \\
\hline CMR mass (g) & $450 \pm 111$ & $301 \pm 93$ & $149 \pm 46$ \\
$\begin{array}{l}\text { Pathology } \\
\text { mass (g) }\end{array}$ & $445 \pm 116$ & $313 \pm 96$ & $128 \pm 40$ \\
R Value & 0.99 & 0.95 & 0.77 \\
\hline
\end{tabular}

$0.9513 \times+29.172 \quad(\mathrm{r}=0.95) ; \mathrm{y}=0.671 \times+28.881 \quad(\mathrm{r}=0.77)$ regressed the total mass, LV mass and RV mass respectively (Figure 2). The average bias between 3D CMR and pathology measures for total mass, LV mass and RV mass were: $3.0 \mathrm{~g}$ (95\% limits of agreement (LOA) -39 to 46), -16 (-82 to 50$)$, and $19 \mathrm{~g}$ (-41 to 80 ), respectively. The differences between CMR measured mass and pathology mass for all three indices were illustrated in Bland-Altman plots (Figure 3).

The intraclass correlation coefficient (ICC) was calculated for ten randomly selected hearts (15\%) to assess intra-observer reliability between measures of 3D MRI volumes made by a single rater using the ATMT method. For 3D MRI total volume measured in October
2013 versus the February 2014 measurement was 0.990 (95\% confidence interval, 0.958 to 0.998 ). For 3D MRI LV volume the ICC measurement was 0.986 (95\% confidence interval, 0.948-0.997). For 3D MRI RV volume the ICC measurement was 0.730 (95\% confidence interval, 0.234-0.925).

\section{Discussion}

The ideal method for quantification of ventricular mass should be fairly quick, non-invasive, highly accurate and reproducible and applicable to each ventricle. In our study, we were able demonstrate that total ventricular mass, LV and RV mass obtained by CMR showed excellent correlation with histopathology mass obtained on the explanted hearts $(r=0.99,0.95$, and 0.77 , respectively). This study demonstrates that accurate and reproducible assessment of both left and right ventricular masses can be efficiently obtained non-invasively using CMR's SSFP sequences, as compared to weighed, explanted human donor hearts. Most importantly, this is the final validation step so critical in unequivocally depicting CMR as the true 'gold standard' that heretofore had been
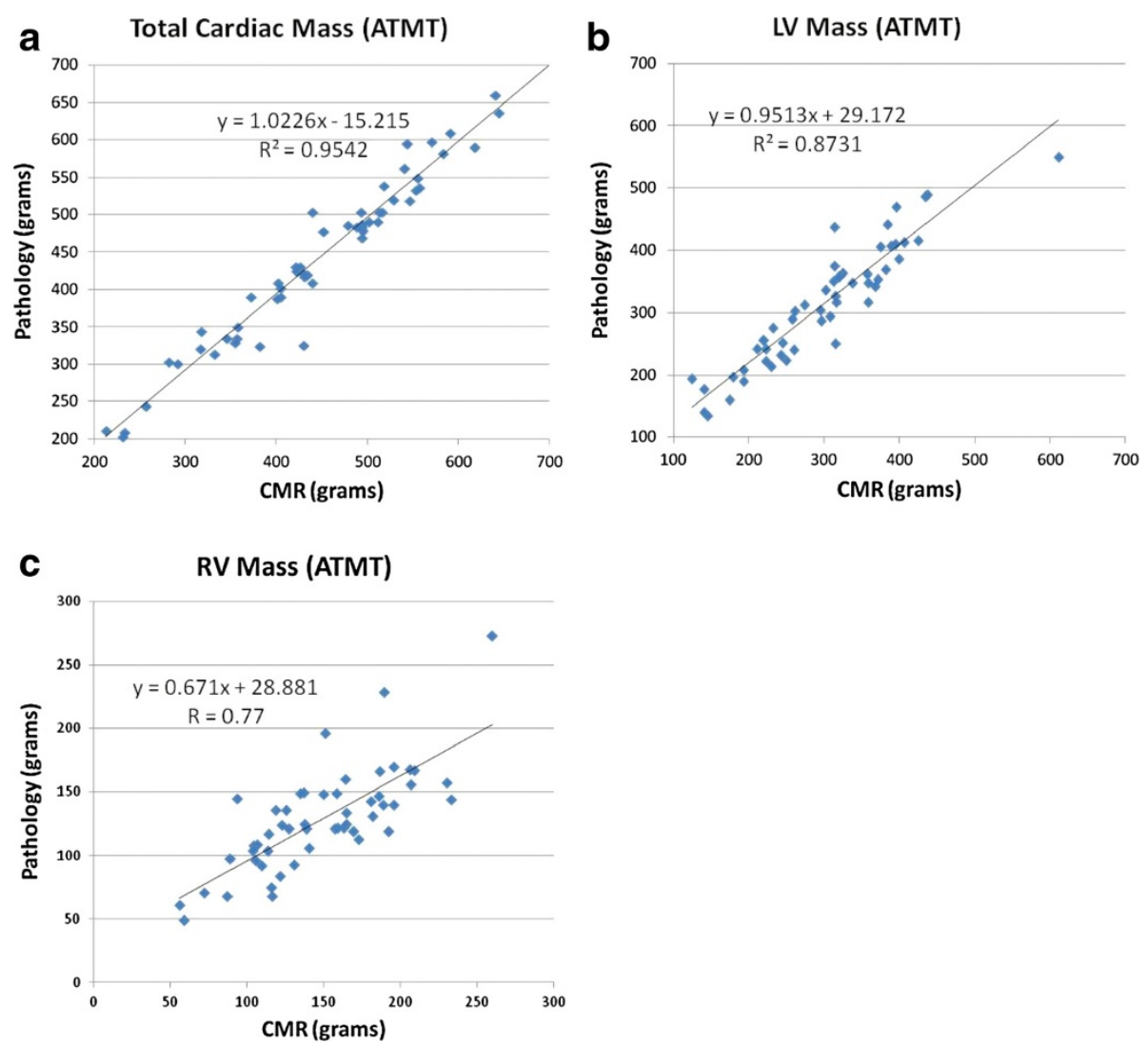

Figure 2 Linear Regression Analysis CMR vs. Pathology Mass. a: comparison of combined ventricular, left and right ventricular mass measurement by cardiac magnetic resonance imaging with autopsy/pathology mass. b: Linear regression analysis: comparison of left ventricular (LV) mass measurement by cardiac magnetic resonance imaging with autopsy/pathology mass. c: Linear regression analysis: comparison of right ventricular (RV) mass measured by cardiac magnetic resonance imaging with autopsy/pathology mass. 
remarkably accepted de facto given the distinct and glaring absence of such a validation step.

Considered not possible in the current era, a confirmation similar to the manner in which Devereux and Reichek first validated echocardiographic mass (Penn Equation) required a resourceful contemporary approach. Awaiting a person's imminent death is considered taboo by stateside IRB's and justifiably so. Arriving on a natural ready supply of explanted ex vivo hearts, while not in vivo, was the crucial element and an aspect that fundamentally required an exceptional synchronization amongst the Cardiac Transplant Service, Pathology and CMR Laboratory at any time. These invaluable results were obtained despite several possibly obfuscating variables. Chiefly, the cardiomyopathic, ex-planted hearts all possessed variable mechanical instrumentation before transplant, including left and right ventricular assist devices (LVAD/RVAD) and implantable cardioverter-defibrillators (AICD) and/or pacemakers. The removal of this instrumentation upon explanation often led to partial or full apical core removal, leaving an apical interface that was far more difficult to assess when drawing epicardial and endocardial contours (Figure 4). As well, a separate lateral wall surgical section was always removed for Surgical Pathology for confirmation of etiology of the cardiomyopathy. Thus, there was a substantial inherent destruction and radical distortion of the primary variable, potentially leading to wholesale inability to achieve reliable correlation. The fact that the whole heart, left and right ventricular masses still correlated extremely well with the weighed masses is a further testament to the accuracy of CMR; an even higher correlation is predicted when the natural boundaries of the heart, with the apex fully intact, provide an enhanced guide for contouring as in routine CMR imaging. A final factor to consider is the use of a cardiomyopathic patient population in this study. Ideally, a separate subgroup of non-cardiomyopathic hearts to establish a second correlation between CMR-derived and weighed ventricular LV and RV mass would be ideal. However, the use of less diseased, non- cardiomyopathic hearts would prove less problematic for epicardial and endocardial contouring, which would likely improve the accuracy of the CMR mass quantitation.

Establishing the accuracy and reproducibility of CMR measured cardiac mass has important implications for clinical practice and research. Prognostic significance of left ventricular mass regression was demonstrated by showing fewer rates of clinical end points in patients with lower LV mass during antihypertensive treatment [18]. An increase in left ventricular mass predicts a higher incidence of cardiovascular events including death and this information is in addition to that provided by the evaluation of traditional cardiovascular risk factors [19]. Thus, accurate measurement of LV mass by CMR has important clinical implications in terms of risk assessment and regression of LV mass in response to treatment. Interestingly, there are no studies in the literature that directly compared RV mass with prognosis. Our study further adds to the literature in demonstrating strong correlation between CMR measured RV mass and pathology mass. We believe this study provides a steppingstone for the use of RV mass as a prognostic stool as well as for assessing response to treatment in patients with pulmonary hypertension.

In a landmark paper, Bottini et al. demonstrated that detecting a 10 gram regression in LV mass with a power of 0.8 and $\mathrm{p}<0.05$ would require 550 patients using echocardiography but only require 17 patients using CMR. Thus, much smaller sample sizes can be used to detect the same change in LV mass, or smaller degrees of changes can be identified using the same sample size. Moreover, the precise measurement of ventricular mass has been proposed to more easily be utilized in a therapeutic approach, such as in LV mass regression with antihypertensive therapy [20]. The implications are important in that therapeutic efficacy, risk stratification and general accuracy can only be obtained when an exacting method is available. In this study, linear regression analysis indicated excellent correlations as shown in Figure 2 for all three indices including total cardiac mass, LV mass and RV mass determined by using 3D CMR with ATMT technique and true LV mass obtained at autopsy on explanted hearts. The differences between CMR measured mass and pathology mass including systemic and random differences for all three indices were illustrated in the Bland-Altman plots (Figure 3). The bias between CMR measured mass and pathology mass were low and not systematic.

An important and controversial consequence of this work has emerged. Namely does the conventional long accepted dogma for cardiac mass quantification excluding certain mass (papillary and trabecular mass) need to be discarded? It is well known that papillary muscles have the same thickness as the left ventricular free wall. Additionally, in patients with LV hypertrophy there is a proportional hypertrophy of the papillary muscles [21]. In a study by Francois, et al [14] it was demonstrated that the most accurate estimates of the LV mass in animal models were obtained when the papillary muscles were included in the myocardium. Furthermore, when the papillary muscles were excluded from the LV mass, the values were significantly lower than pathology mass measured at autopsy. However, historically, it is interesting to note that in the initial validation of GRE the correlations were improved by excluding the papillary muscles and fine trabecular tissue, no doubt due to the well-known flow-related enhancement that occurred with GRE causing overestimation, not underestimation. This then triggered the venerable mathematical regression correction we now use today. Interestingly, despite 


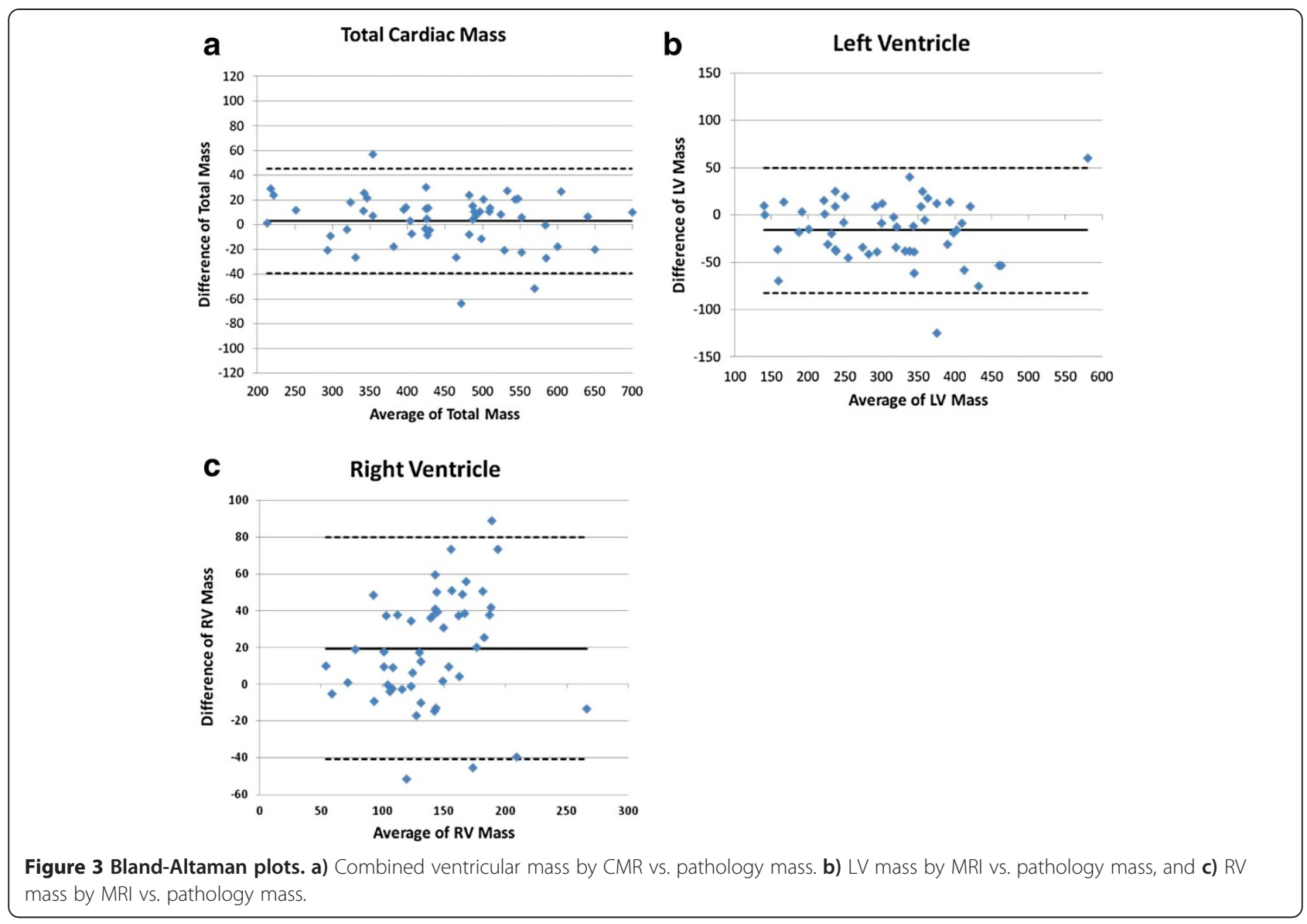

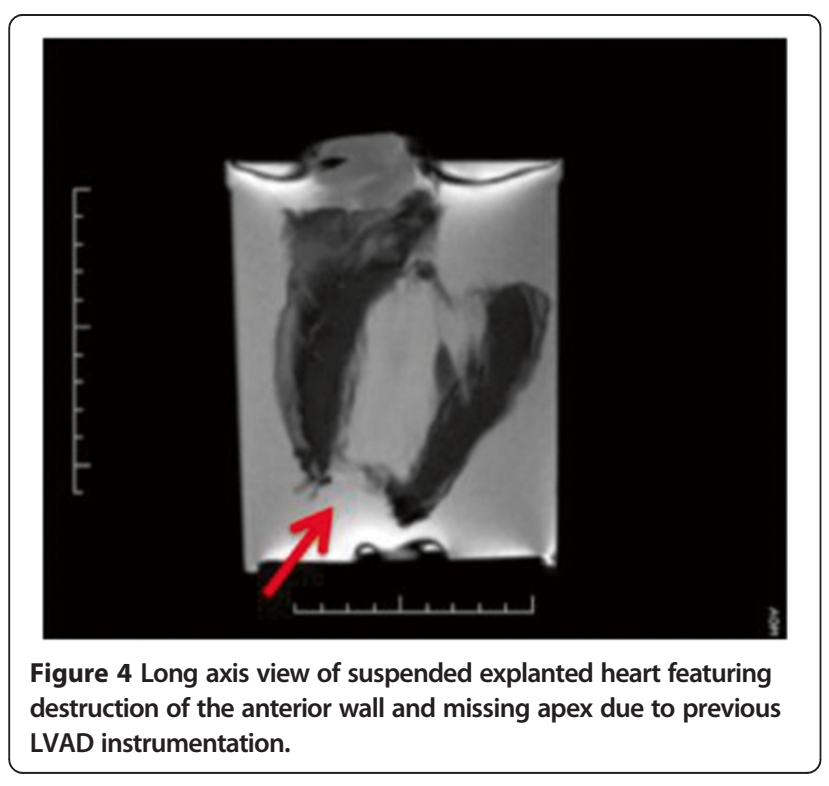

this knowledge, the modern and long-lauded SSFP sequences, devoid of this artifact, has not sparked a plea for reconsideration of the general approach of excluding papillary and trabecular tissue. Similar to the previous SSFP animal studies, our study incorporated papillary muscles and trabecular mass by using the ATMT method in calculating total LV mass while obtaining near unity with true autopsy mass. Further, the new regression equation now includes, not excludes, papillary and trabecular mass suggesting that our Society should now incorporate both into our manual contouring approaches. In conclusion, to our knowledge this study represents the first human modern CMR versus autopsy validation of human ventricular mass, and fairly emulates the intrepid days of the initial validation of the Penn Formula by Devereux and Reichek in 1977.

\section{Study limitations}

Our chief limitation is that CMR scans were performed on ex-vivo hearts that were suspended in normal saline and these specimens obligatorily do not suffer from temporal resolution issues encountered in in-vivo, live, beating hearts. The contribution of this temporal blur is 
felt to be far less than the inexactitude of the underestimation encountered by systematically excluding important myocardium. It should also be noted that the initial premise of our study was to image patients referred for heart transplant via CMR, but as our center is a large tertiary/quaternary referral base, only the rare patient presented without an AICD. None of the hearts in our study had normal pathology and, in addition, significant numbers of explanted hearts (98\%) possessed variable mechanical instrumentation before transplantation (e.g. LVADs, RVADs, and AICDs) which confounded natural contouring. The removal of this instrumentation upon explanation often led to partial or full apical (often biventricular) core removal, leaving an apical interface that was more difficult to assess when drawing epicardial and endocardial contours. Yet, despite the high prevalence of patients with prior mechanical instrumentation and the attendant difficulties of imaging these hearts, we still found excellent correlations between CMR and autopsy myocardial mass which supports the value of our approach. Further, that the RV correlations were less than the LV correlations might be expected due to the disproportionate effect of loss of RV contours with surgical manipulation. Less expected however was the effect in which the now deflated heart post-explant lost its RV/ septal margins making separation of RV and LV less precise. In that the whole heart demonstrates near identity with autopsy weight prior to separation attests to the fidelity of the approach mitigating the absolute process itself. Finally, explanted hearts in this study were imaged at higher resolution than in-vivo hearts. For the purpose of the study, a limited separate analysis showed no significant difference when measured at conventional resolution.

\section{Conclusions}

SSFP-CMR accurately determines total myocardial, LV and $\mathrm{RV}$ masses as compared to weighed explanted hearts, despite variable surgical removal of instrumentation (LVAD/ RVAD, AICD's and often apical core removals) in a transplant population. Thus, while GRE was the original 'gold standard' for LV mass, SSFP, despite its current universal acceptance as the 'de facto' gold standard', is now formally validated in this autopsy study. Further, the regression equation now includes, not excludes, papillary and trabecular mass supporting the notion that our Society should consider incorporating both into standard cardiac measurements.

\footnotetext{
Abbreviations

AICD: Automatic implantable cardioverter defibrillator; ATMT: Automatic Thresholding with Manual Trimming; CMR: Cardiovascular magnetic resonance imaging; GRE: Gradient-recalled echo; ICC: Intra-class correlation coefficient; LV: Left ventricle; LVAD: Left ventricular assist device; LVH: Left ventricular hypertrophy; LVM: Left ventricular mass; PM: Pacemaker; RV: Right ventricle; RVAD: Right ventricular assist device; RVM: Right ventricular mass; SSFP: Steady-state free precession.
}

Competing interests

All the authors declare that they have no competing interests.

\section{Authors' contribution}

NF involved in the design and first draft of manuscript; SR participated in the specimen collection, analysis and manuscript writing; MD participated in the design, data and image analysis and manuscript writing; GR involved in software analysis; DT participated in statistical analysis; PO and JG were involved pathology specimen analysis; RW and JY were involved in specimen collection and imaging; SM participated in transplant considerations, RB originated the research concept, was involved in the design, data analysis, manuscript writing, editing, approved final manuscript and takes responsibility for the published manuscript.

\section{Authors' information}

Robert WW Biederman, MD, FACC, FAHA, FSGC, FASA

Associate Professor of Medicine, Cardiovascular Magnetic Resonance

Imaging, Temple University, College of Medicine, Gerald McGinnis Cardiovascular Institute

Adjunct Associate Professor of Bioengineering \& Fluid Mechanics, Carnegie Mellon University, Allegheny General Hospital

320 East North Avenue, Pittsburgh, Pennsylvania 15212, USA

\section{Acknowledgements}

The senior author is indebted to innumerable conversations over the years with Nat Reichek and Richard Devereux.

Presented in part at SCMR, Nice, France 2011 and AHA 2012.

\section{Author details}

${ }^{1}$ Gerald McGinnis Cardiovascular Institute, Allegheny General Hospital, Pittsburgh, PA, USA. 'Department of Pathology, Allegheny General Hospital, Pittsburgh, PA, USA.

Received: 27 February 2014 Accepted: 28 August 2014

Published online: 01 October 2014

\section{References}

1. Myerson SG, Bellenger NG, Pennell DJ. Assessment of left ventricular mass by cardiovascular magnetic resonance. Hypertension. 2002; 39(3):750-5.

2. Alfakih K, Bloomer T, Bainbridge S, Bainbridge G, Ridgway J, Williams G, Sivananthan M. A comparison of left ventricular mass between two-dimensional echocardiography, using fundamental and tissue harmonic imaging, and cardiac MRI in patients with hypertension. Eur J Radiol. 2004; 52:103-9.

3. Bottini PB, Carr AA, Prisant LM, Flickinger FW, Allison JD, Gottdiener JS. Magnetic resonance imaging compared to echocardiography to assess left ventricular mass in the hypertensive patient. Am J Hypertens. 1995; 8:221-8.

4. Missouris CG, Forbat SM, Singer DR, Markandu ND, Underwood R, MacGregor GA. Echocardiography overestimates left ventricular mass: a comparative study with magnetic resonance imaging in patients with hypertension. J Hypertens. 1996; 14:1005-10.

5. Bellenger NG, Marcus NJ, Rajappan K, Yacoub M, Banner NR, Pennell DJ. Comparison of techniques for the measurement of left ventricular function following cardiac transplantation. J Cardiovasc Magn Reson. 2002; 4(2):255-63.

6. Bellenger NG, Burgess MI, Ray SG, Lahiri A, Coats AJ, Cleland JG, Pennell DJ. Comparison of left ventricular ejection fraction and volumes in heart failure by echocardiography, radionuclide ventriculography and cardiovascular magnetic resonance; are they interchangeable? Eur Heart $\rfloor$ 2000; 21(16):1387-96.

7. Bellenger NG, Davies LC, Francis JM, Coats AJ, Pennell DJ. Reduction in sample size for studies of remodeling in heart failure by the use of cardiovascular magnetic resonance. J Cardiovasc Magn Reson. 2000; 2(4):271-8.

8. Childs H, Ma L, Ma M, Clarke J, Cocker M, Green J, Strohm O, Friedrich MG. Comparison of long and short axis quantification of left ventricular volume parameters by cardiovascular magnetic resonance, with ex-vivo validation. J Cardiovasc Magn Reson. 2011; 13:40. 
9. Strohm O, Schulz-Menger J, Pilz B, Osterziel KJ, Dietz R, Friedrich MG Measurement of left ventricular dimensions and function in patients with dilated cardiomyopathy. J Magn Reson Imaging. 2001; 13(3):367-71.

10. Hung J, Lang R, Flachskampf F, Shernan SK, McCulloch ML, Adams DB, Thomas J, Vannan M, Ryan T. ASE. 3D echocardiography: a review of the current status and future directions. J Am Soc Echocardiogr. 2007; 20:213-33.

11. Devereux RB, Reichek N. Echocardiographic determination of left ventricular mass in man. Anatomic validation of the method. Circulation. 1977; 55:613-8.

12. Feino DS, Jaffe WC, Simonetti OP, Judd RM, Finn JP. TrueFSIP: assessment of accuracy for measurement of left ventricular mass in an animal model. J Magn Reson Imaging. 2002; 15:526-31.

13. Shors SM, Fung CW, François CJ, Finn JP, Fieno DS. Accurate quantification of right ventricular mass at MR imaging by using cine true fast imaging with steady-state precession: study in dogs. Radiology. 2004; 230:383-8.

14. François CJ, Fieno DS, Shors SM, Finn JP. Left ventricular mass: manual and automatic segmentation of true FISP and FLASH cine MR images in dogs and pigs. Radiology. 2004; 230:389-95.

15. Franco F, Dubois SK, Peshock RM, Shohet RV. Magnetic resonance imaging accurately estimates LV mass in a transgenic mouse model of cardiac hypertrophy. Am J Physiol. 1998; 274:H679-83.

16. Stephensen SS, Carlsson M, Ugander M, Engblom H, Olivecrona G, Erlinge D, Arheden $\mathrm{H}$. Agreement of left ventricular mass in steady state free precession and delayed enhancement MR images: implications for quantification of fibrosis in congenital and ischemic heart disease. BMC Med Imaging. 2010; 10:4.

17. Portney LG, Watkins MP. Foundations of clinical research: Applications to practice (3rd edition) Jan 10; 2008.

18. Devereux RB, Wachtell K, Gerdts E, Bowman K, Nieminen MS, Papademetrou V, Rokkedal J, Harris K, Aurup P, Dahlof B. Prognostic significance of left ventricular mass change during treatment of hypertension. JAMA. 2004; 292:2350-6.

19. Levy D, Garrison RJ, Savage DD, Kannel WB, Castelli WP. Prognostic implications of echocardiographically determined left ventricular mass in the Framingham Heart Study. N Engl J Med. 1990; 322:1561-6.

20. Nadour W, Biederman RW. Is left ventricular hypertrophy regression important? Does the tool used to detect it matter? I Clin Hypertens. 2009; 11(8):441-7.

21. Estes EH, Dalton FM, Entman MI, Dixon HB 2nd, Hackel DB. The anatomy and blood supply of the papillary muscles of the left ventricle. Am Heart $\mathrm{J}$. 1966; 71:356-62.

doi:10.1186/s12968-014-0074-0

Cite this article as: Farber et al.: Ex vivo cardiovascular magnetic resonance measurements of right and left ventricular mass compared with direct mass measurement in excised hearts after transplantation: a first human SSFP comparison. Journal of Cardiovascular Magnetic Resonance 2014 16:74.

\section{Submit your next manuscript to BioMed Central and take full advantage of:}

- Convenient online submission

- Thorough peer review

- No space constraints or color figure charges

- Immediate publication on acceptance

- Inclusion in PubMed, CAS, Scopus and Google Scholar

- Research which is freely available for redistribution 